\title{
Electrocardiogram Signal Denoising Using Discrete Wavelet Transform
}

\author{
Mustapha El HANINE ${ }^{1}$, Elhassane Abdelmounim ${ }^{1}$,Rachid Haddadi ${ }^{1}$ and Abdelaziz Belaguid ${ }^{2}$ \\ 1 Faculty of Science and Technology, University of Hassan I, Settat 26000 Morocco \\ 2 Faculty of Medicine and Pharmacy, University of Mohamed V, Rabat 10000 Morocco
}

\begin{abstract}
The most common noises in ECG (electrocardiogram) signal processing are BW (baseline wandering) and the 50 or $60 \mathrm{~Hz}$ PLI (power line interferences). In order to remove these two major source of noises, we have used the recent powerful DWT (discrete wavelet transform) signal processing in ECG signals which are obtained from MIT-BIH Arrhythmia Database. The results indicate that DWT is a good method for filtering noises without changing the morphology of ECG, and can be applied to all types of ECG signals, whether normal or presenting arrhythmias.
\end{abstract}

Key words: ECG, Signal processing, $60 \mathrm{~Hz}$ PLI, baseline wander, DWT.

\section{Introduction}

Muscular contraction is associated with electrical changes known as depolarization. The ECG is a measure of this electrical activity associated with the heart. The ECG is measured at the body surface and results from electrical changes associated with activation first of the two small heart chambers, the atria, and then of the two larger heart chambers, the ventricles. Analysis of the local morphology of the ECG signal and its time varying properties has produced a variety of clinical diagnostic tools. It is also an essential tool to allow monitoring patients at home, thereby advancing telemedicine applications.

Producing an algorithm for the detection of the $\mathrm{P}$ wave, QRS complex and T wave in an ECG is a difficult problem due to the time varying morphology of the signal subject to physiological conditions and the presence of noise. There are several types of noises that affect the ECG. The BW (baseline wander) and 50 or $60 \mathrm{~Hz}$ interferences are some of these types. The goal of ECG enhancement is to separate the valid ECG from the undesired artifacts so as to present a

Corresponding author: Mustapha El Hanine, professor, research fields: digital signal processing, filtering and compression. E-mail: melhanine@yahoo.fr. signal that allows easy visual interpretation [1].

\section{ECG Wave Pattern}

\subsection{ECG Signal}

ECG is a recording of biopotential signal that is generated by electrical cardiac activity and it is used by clinicians to identify various heart diseases such as myocardial infarction, conduction defects and arrhythmia. ECG signal consists of a well defined successive set of: $\mathrm{P}$ wave, PQ interval (or PR), QRS complex, ST segment and T wave.

The $\mathrm{P}$ wave represents the depolarization of left and right atria that generates the contraction of the atria and the ejection of blood to the ventricles. The QRS segment represents the depolarization of the left and right ventricles that generates the contraction of the ventricles and the ejection of blood to the aorta and pulmonary artery. The $\mathrm{T}$ wave represents the period of time when the ventricles repolarize. Fig. 1 shows a typical ECG signal.

\subsection{Power Spectrum of the ECG}

The ECG waveform contains, in addition to the QRS complex, $\mathrm{P}$ and $\mathrm{T}$ waves, $60 \mathrm{~Hz}$ noise from power line interferences, EMG from muscles, motion 


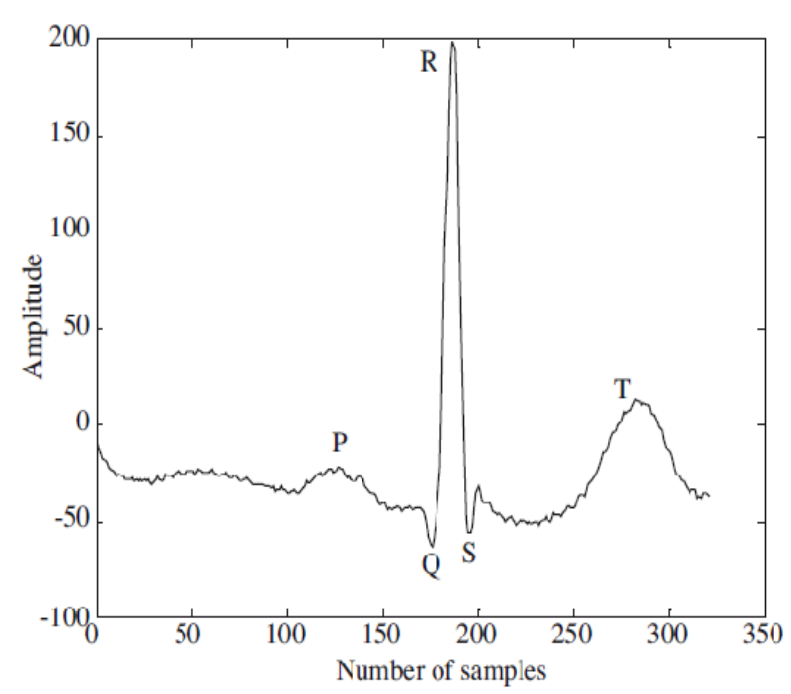

Fig. 1 Typical ECG signal with P-wave, QRS complex, and $\mathrm{T}$-wave (sampling frequency is $360 \mathrm{~Hz}$ ).

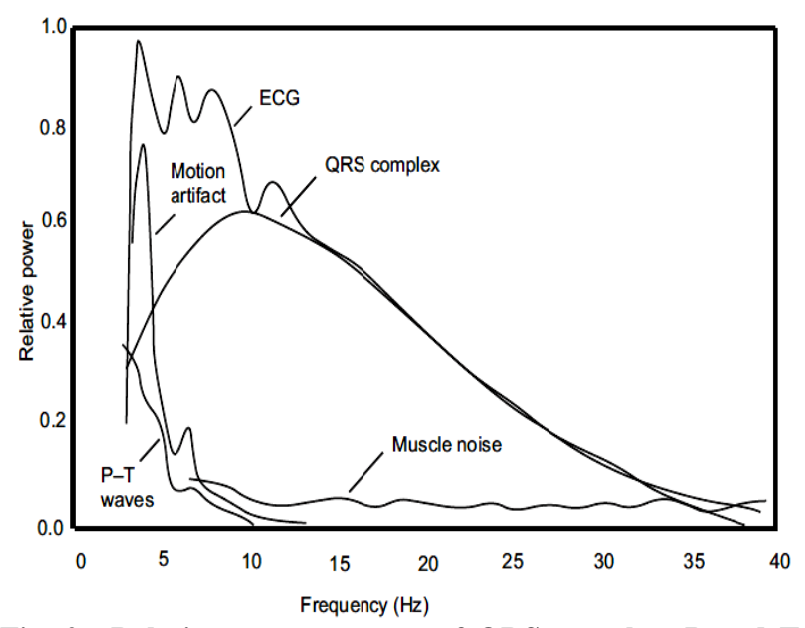

Fig. 2 Relative power spectra of QRS complex, $P$ and $T$ waves, muscle noise and motion artifacts based on an average of 150 beats.

artifact from the electrodes and skin interfaces, and possibly other interferences.

Fig. 2 summarizes the relative power spectrum of the ECG, QRS complexes, $\mathrm{P}$ and $\mathrm{T}$ waves, motion artifact, and muscle noise [2].

The recorded ECG signal is often contaminated by different types of noises and artifacts that can be within the frequency band of ECG signal, which may change the characteristics of ECG signal. Hence it is difficult to extract useful information of the signal. The corruption of ECG signal is due to following major noises [3-5].

\subsection{Baseline Wander}

BW of ECG signals is usually caused by respiration or movement of the subject and appears as a low frequency artifact. The removal of this disturbance is an important step in ECG signal analysis, not only to produce a stable signal for subsequent automatic processing, but also for reliable visual interpretation. As the frequency of ECG signals varies with time, using an ordinary high-pass filter can distort the waveform. As an alternative, an improved method involving DWT is proposed.

\subsection{Power Line Interferences}

Power line interferences contains $60 \mathrm{~Hz}$ pickup (in USA) or $50 \mathrm{~Hz}$ pickup (in Morocco) because of improper grounding. It is indicated as an impulse or spike at $60 \mathrm{~Hz} / 50 \mathrm{~Hz}$ harmonics, and will appear as additional spikes at integral multiples of the fundamental frequency. Its frequency content is 60 $\mathrm{Hz} / 50 \mathrm{~Hz}$ and its harmonics, amplitude is up to 50 percent of peak-to-peak ECG signal amplitude. A 60 $\mathrm{Hz}$ notch filter can be used to remove the power line interferences, but in this work the proposed method using DWT yields good results.

\section{Wavelet Transform}

The wavelet transform has emerged over recent years as a key time-frequency analysis and coding tool for the ECG. Its ability to separate out pertinent signal components has led to a number of wavelet-based techniques which supersede those based on traditional Fourier methods. In its continuous form, the CWT allows a powerful analysis of non-stationary signals, making it ideally suited for the high-resolution interrogation of the ECG over a wide range of applications. In its discrete form, the DWT provides the basis of powerful methodologies for partitioning pertinent signal components which serve as a basis for potent compression strategies. 


\subsection{Continuous Wavelet Transform}

The CWT transforms a continuous signal into highly redundant signal of two continuous variables: translation and scale. The resulting transformed signal is easy to interpret and is valuable for time-frequency analysis. The continuous wavelet transform of continuous function, $\mathrm{x}(t)$ relative to real-valued wavelet, $\psi(t)$ is described by:

$$
W_{\Psi}(s, \tau)=\int_{-\infty}^{+\infty} x(t) \psi_{s, \tau}^{*}(t) d t
$$

where,

$$
\psi_{s, \tau}(t)=\frac{1}{\sqrt{s}} \psi\left(\frac{t-\tau}{s}\right)
$$

$\mathrm{s}$ and $\tau$ are called scale and translation parameters, respectively. $\mathrm{W}_{\psi}(s, \tau)$ denotes the wavelet transform coefficients and $\psi$ is the fundamental mother wavelet.

\subsection{Discrete Wavelet Transform}

The DWT has become a powerful technique in biomedical signal processing. It can be written on the same form as Eq. (1), which emphasizes the close relationship between CWT and DWT. The most obvious difference is that the DWT uses scale and position values based on powers of two.The values of $s$ and $\tau$ are: $s=2^{j}, \tau=k^{*} 2^{j}$ and $(j, k) \in Z^{2}$ as shown in Eq. (3).

$$
\psi_{s, \tau}(t)=\frac{1}{\sqrt{2^{j}}} \psi\left(\frac{t-k * 2^{j}}{2^{j}}\right)
$$

The key issues in DWT and inverse DWT are signals decomposition and reconstruction, respectively. The basic idea behind decomposition and reconstruction is low-pass and high-pass filtering with the use of down sampling and up sampling, respectively. The result of wavelet decomposition is hierarchically organized decompositions. One can choose the level of decomposition $j$ based on a desired cutoff frequency. Fig. 3a shows an implementation of a three-level forward DWT based on a two-channel recursive filter bank, where $\mathrm{h}_{0}(n)$ and $\mathrm{h}_{1}(n)$ are low-pass and high-pass analysis filters, respectively, and the block $\downarrow 2$ represents the down sampling operator by a factor of 2 . The input signal $\mathrm{x}(n)$ is recursively decomposed into a total of four subband signals: a coarse signal $\mathrm{C}_{3}(n)$, and three detail signals, $\mathrm{d}_{3}(n), \mathrm{d}_{2}(n)$, and $\mathrm{d}_{1}(n)$, of three resolutions. Fig. $3 b$ shows an implementation of a three-level inverse DWT based on a two-channel recursive filter bank, where $\widetilde{h}_{0}(n)$ and $\tilde{h}_{1}(n)$ are low-pass and high-pass synthesis filters, respectively, and the block $\uparrow 2$ represents the up sampling operator by a factor of 2 . The four subband signals $\mathrm{C}_{3}(n), \mathrm{d}_{3}(n)$, $\mathrm{d}_{2}(n)$ and $\mathrm{d}_{1}(n)$ are recursively combined to reconstruct the output signal $\widetilde{x}(n)$. The four finite impulse response filters satisfy the following relationships:

$$
\begin{gathered}
h_{1}(n)=(-1)^{n} h_{0}(L+1-n) \\
\widetilde{h}_{0}(n)=h_{0}(L+1-n) \\
\widetilde{h}_{1}(n)=(-1)^{n-1} h_{0}(L+1-n)
\end{gathered}
$$

where, $L$ is the length of filters, and $n=1,2, \ldots, L$.

So that the output of the inverse DWT is identical to the input of the forward DWT $[6,7]$.

\section{Methodology}

In the simulation studies, test ECG signals came from MIT-BIH Arrhythmia Database. They concern leads V1 and D2, and they were digitized at 360 samples per second with 11 bit resolution over \pm 5 $\mathrm{mV}$ range. Sample values thus ranged from 0 to 2,047
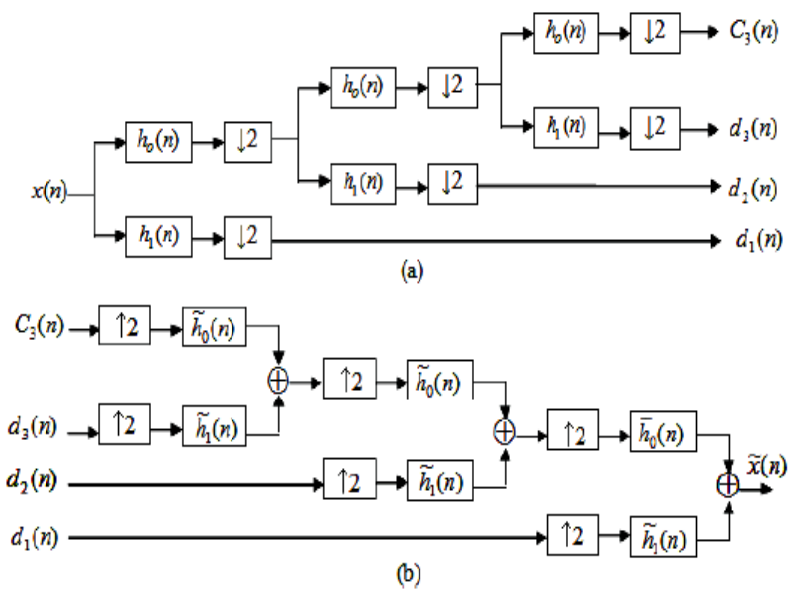

Fig. 3 A three-level two-channel iterative filter bank: (a) forward DWT; (b) inverse DWT. 
inclusive, with a value of 1,024 corresponding to 0 $\mathrm{mV}[8]$

\subsection{Baseline Wander}

Baseline drift having a frequency range of $(0 \mathrm{~Hz}, \ldots, 0.5 \mathrm{~Hz})$. In accordance with Nyquist's rule, if the original signal has a highest frequency $f_{\max }$, it requires a sampling frequency $f_{s} \geq 2 f_{\max }$. Hence, at each decomposition level $j$, the frequency axis is recursively divided into halves at the ideal cut-off frequencies $\mathrm{f}_{j}=$ $f_{\max } / 2^{j}[9]$.

The ECG records taken from the MIT-BIH arrhythmia database are sampled at $360 \mathrm{~Hz}\left(f_{s}=\right.$ $360 \mathrm{~Hz}$ ). The maximum frequency is on the order of $130 \mathrm{~Hz}\left(f_{\max }=130 \mathrm{~Hz}\right)[9,10]$. Therefore, the range of real frequency components of the signals is between 0 $\mathrm{Hz}$ and $130 \mathrm{~Hz}$. The correspondence between DWT coefficients and range of frequencies is given inTable1.

The proposed method for cancelling the $\mathrm{BW}$ is based on wavelet decomposition up to level 8, which generates a set of approximation coefficients(C8), and eight sets of detail coefficients $(d 1, \ldots, d 8)$. By cancellation of approximations, the filtered signal is recovered from the details only. This is equivalent to a high-pass filter cutoff frequency $f_{c}=f_{\max } / 256$.

Fig.4 shows an example of the BW removal. The original ECG signal has low-frequency fluctuations; after removing it, the filtered ECG signal appears centered around a horizontal line.

\subsection{Power Line Interferences}

The ECG signals from the database MIT-BIH are affected by $60 \mathrm{~Hz}$ PLI. This noise was filtered with an analog notch filter, but its influence still appears. We propose, therefore, to add to the test signals, a simulated noise of this form:

$$
n(t)=A * \sin \left(2 \pi f_{0} t\right)
$$

where, $A$ is the amplitude, and $f_{0}$ is the $60 \mathrm{~Hz}$ frequency of the simulated noise.

The noisy signal may then be expressed by:
Table 1 Range frequencies of DWT coefficients.

\begin{tabular}{ll}
\hline DWT coefficients & Range frequencies \\
\hline d1 & $65 \mathrm{~Hz}, \ldots, 130 \mathrm{~Hz}$ \\
d2 & $32.5 \mathrm{~Hz}, \ldots, 65 \mathrm{~Hz}$ \\
d3 & $16.25 \mathrm{~Hz}, \ldots, 32.5 \mathrm{~Hz}$ \\
d4 & $8.125 \mathrm{~Hz}, \ldots, 16.25 \mathrm{~Hz}$ \\
d5 & $4.062 \mathrm{~Hz}, \ldots, 8.125 \mathrm{~Hz}$ \\
d6 & $2.031 \mathrm{~Hz}, \ldots, 4.062 \mathrm{~Hz}$ \\
d7 & $1.015 \mathrm{~Hz}, \ldots, 2.031 \mathrm{~Hz}$ \\
d8 & $0.507 \mathrm{~Hz}, \ldots, 1.015 \mathrm{~Hz}$ \\
$\mathrm{C} 8$ & $0 \mathrm{~Hz}, \ldots, 0.507 \mathrm{~Hz}$ \\
\hline
\end{tabular}

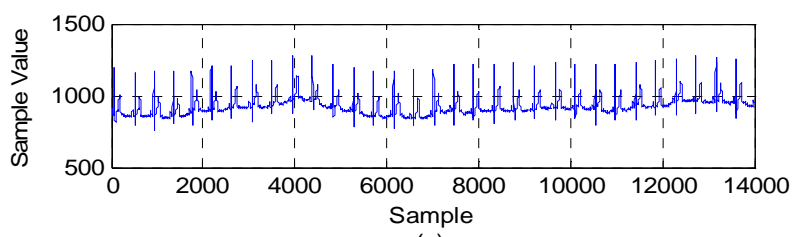

(a)

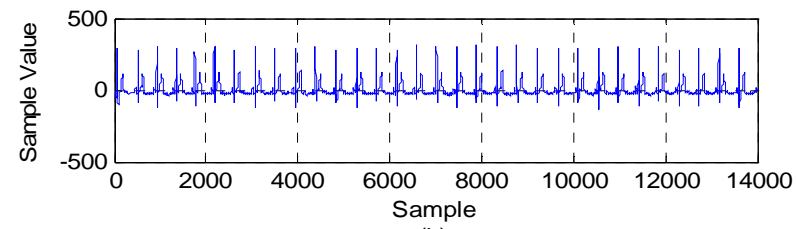

(b)

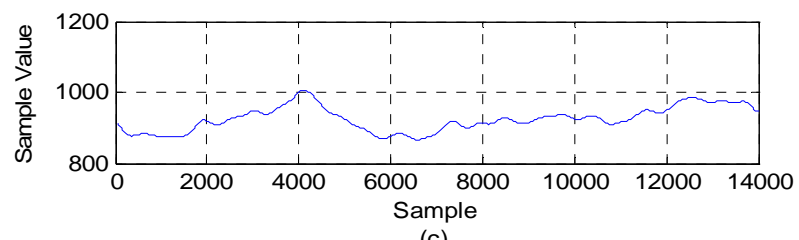

Fig. 4 ECG 117.dat [10000...24000]: (a) noised ECG with BW; (b) filtrered ECG; (c) removed baseline wander.

$$
x(t)=\operatorname{ecg}(t)+n(t)
$$

where, $\operatorname{ecg}(t)$ is the signal from the MIT-BIH database.

The filtering process is done in four steps:

Step 1: At level 1, the signal is decomposed into a set of approximation coefficients $(C l)$, and a set of detail coefficients $(d 1)$.

Step 2: Each set of coefficients of level 1 is decomposed into two other sets (Level 2).

Step 3: The coefficients of level 2 are decomposed into two new sets (Level 3).

Step 4: The filtered signal is reconstructed by removing coefficients containing the $60 \mathrm{~Hz}$ power line noise in their range frequency.

The correspondence between decomposition levels 
and DWT coefficients is given in Table2.

In Table 3, we can see the correspondence between DWT coefficients and range frequencies.

In our case, the denoised signal is recovered from all coefficients except details $d 33(48.75 \mathrm{~Hz}, \ldots, 65$ $\mathrm{Hz})$.

Fig. 5 shows an example of the $60 \mathrm{~Hz}$ power line noise removal.

Figs. 6-8 show the power spectrum density at $60 \mathrm{~Hz}$ of noised ECG (117.dat), filtered ECG and $60 \mathrm{~Hz}$ power line noise. The amplitude $A$ was fixed at $50(A$ $=50)$ respectively.

\subsection{Denoising Evaluation Criteria}

To analyze and evaluate the filtering performance, we used PSD (power spectrum density) [11], percent of COR (cross-corelation coefficient) [12, 13] and PRD (percent of root squared mean difference) as a quantitative criteria.

$$
\begin{gathered}
C O R=100 \cdot \frac{\sum_{n=1}^{N} x(n) \cdot y(n)}{\sqrt{\sum_{n=1}^{N} x^{2}(n) \cdot \sum_{n=1}^{N} y^{2}(n)}} \\
P R D=100 \cdot \sqrt{\frac{\sum_{n=1}^{N}(x(n)-y(n))^{2}}{\sum_{n=1}^{N} x^{2}(n)}}
\end{gathered}
$$

\begin{tabular}{|c|c|c|c|c|c|c|c|c|}
\hline Level & \multicolumn{8}{|c|}{ DWT coefficients } \\
\hline 1 & \multicolumn{4}{|c|}{$\mathrm{C} 1$} & \multicolumn{4}{|c|}{ d1 } \\
\hline 2 & \multicolumn{2}{|c|}{$\mathrm{C} 20$} & \multicolumn{2}{|c|}{$\mathrm{d} 21$} & \multicolumn{2}{|c|}{$\mathrm{d} 22$} & \multicolumn{2}{|c|}{$\mathrm{d} 23$} \\
\hline 3 & $\mathrm{C} 30$ & $\mathrm{~d} 31$ & $\mathrm{~d} 32$ & d33 & d34 & $\mathrm{d} 35$ & $\mathrm{~d} 36$ & d37 \\
\hline
\end{tabular}

Table 2 DWT coefficients of different levels.

Table 3 Range frequencies of DWT coefficients.

\begin{tabular}{ll}
\hline DWT coefficients & Range frequencies \\
\hline C30 & $0 \mathrm{~Hz}, \ldots, 16.25 \mathrm{~Hz}$ \\
d31 & $16.25 \mathrm{~Hz}, \ldots, 32.5 \mathrm{~Hz}$ \\
d32 & $32.5 \mathrm{~Hz}, \ldots, 48.75 \mathrm{~Hz}$ \\
d33 & $48.75 \mathrm{~Hz}, \ldots, 65 \mathrm{~Hz}$ \\
d34 & $65 \mathrm{~Hz}, \ldots, 81.25 \mathrm{~Hz}$ \\
d35 & $81.25 \mathrm{~Hz}, \ldots, 97.5 \mathrm{~Hz}$ \\
d36 & $97.5 \mathrm{~Hz}, \ldots, 113.75 \mathrm{~Hz}$ \\
d37 & $113.75 \mathrm{~Hz}, \ldots, 130 \mathrm{~Hz}$ \\
\hline
\end{tabular}
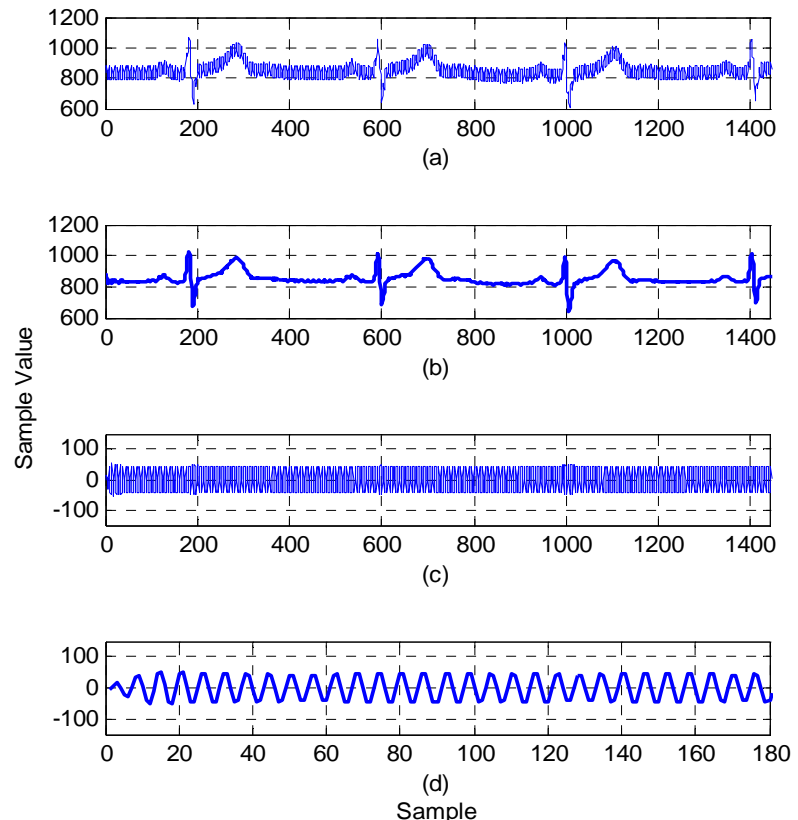

Fig. 5 ECG signal 117.dat [1..1440]: (a) noised ECG; (b) filtred ECG; (c) $60 \mathrm{~Hz}$ power line noise; (d) $60 \mathrm{~Hz}$ noise zoomed in $0.5 \mathrm{~s}$.

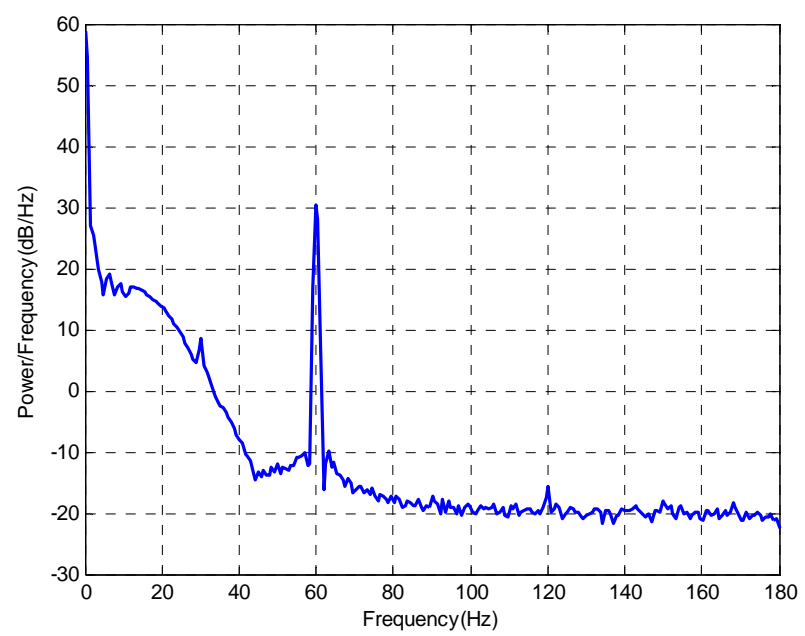

Fig. 6 Power spectrum density of noised ECG 117.dat.

where, $\mathrm{x}(n)$ is the original signal without noises and $\mathrm{y}(n)$ is the filtered signal.

$\mathrm{x}(n)$ is obtained by applying our method in a first step on a ECG records (MIT-BIH) with a natural baseline noise. $\mathrm{y}(n)$ is obtained by applying the method in a second step on $\mathrm{x}(n)$ noised by the natural base line drift or artificial $60 \mathrm{~Hz}$ interferences.

COR reflects the similitude between the two signals. If the two signals are identical, the value of COR is $100 \%$. 


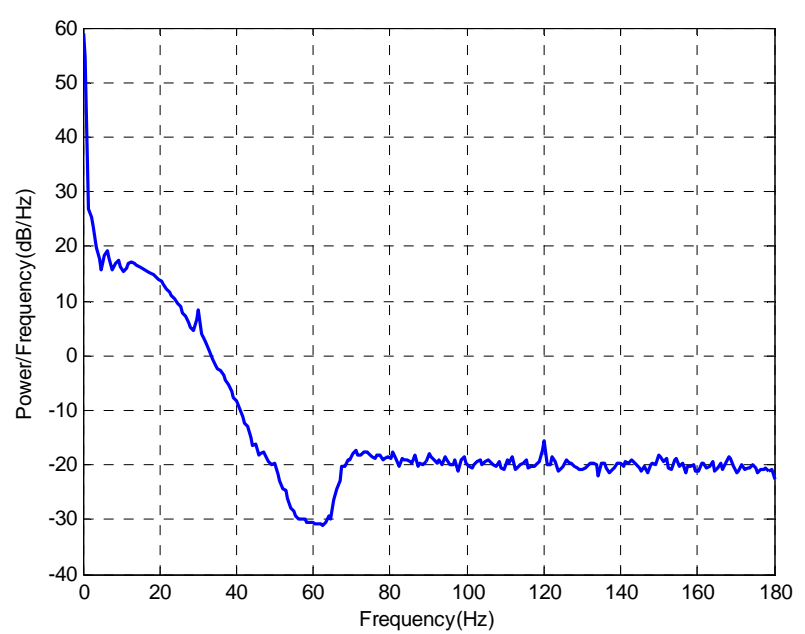

Fig. 7 Power spectrum density of Filtrered ECG 117.dat.

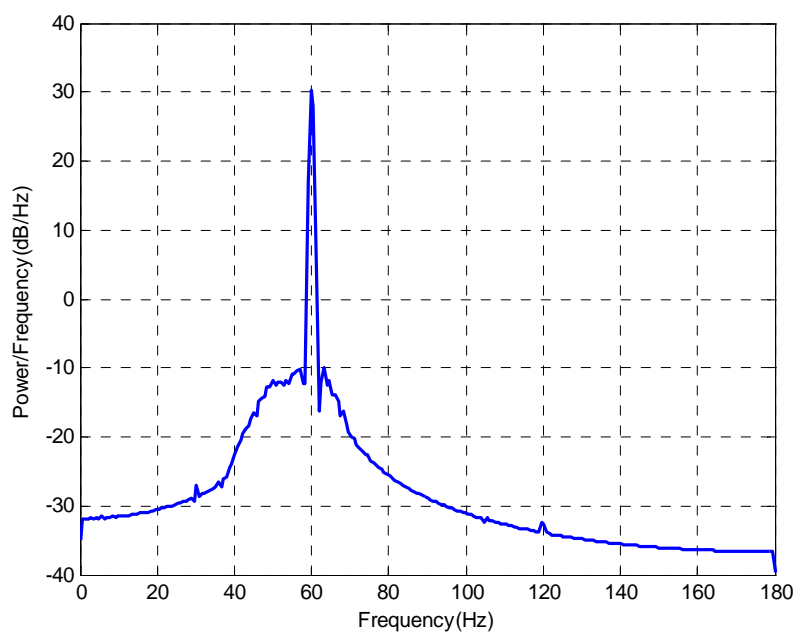

Fig. 8 Power spectrum density of removed $60 \mathrm{~Hz}$ PLI.

PRD reflects the relative distance between the two signals. If they are identical, the value of PRD is $0 \%$.

The PSD is evaluated at $0.5 \mathrm{~Hz}$ for $\mathrm{BW}$, and at 60 $\mathrm{Hz}$ for PLI using Welch method.

\section{Results and Discussions}

After testing several types of wavelets (Symlet, Coiflet, Daubechies,...), the best results are obtained with the Daubechies wavelet db 45.

Table 4 represents the PSD results obtained for five signals from the MIT-BIH database, with a natural baseline drift; it concerns records: 117, 119, 203, 207 and 210 .

Table 5 represents the PSD results for five records, $117,119,203,207$, and 210 with an artificial $60 \mathrm{~Hz}$ noise for different amplitudes.
Table6 represents the COR and PRD criteria for evaluating both baseline wander and $60 \mathrm{~Hz}$ interferences filtering.

The experimental results indicate that this method performs accurate removal $(\mathrm{COR}=100 \%)$ of $\mathrm{ECG}$ BW, while only $92.2 \%$ for median filtering method and $99.6 \%$ for EMD (empirical mode decomposition) correction method [13].

The results presented in Table4 show a significant attenuation of the PSD after filtering. After baseline suppression, all signals are aligned around the horizontal line.

Table 4 PSD criteria for evaluating the baseline suppression.

\begin{tabular}{llll}
\hline \multirow{2}{*}{ ECG } & \multicolumn{3}{c}{ PSD at $0.5 \mathrm{~Hz}(\mathrm{~dB} / \mathrm{Hz})$} \\
\cline { 2 - 4 } & Before filtering & After filtering & Attenuation \\
\hline 117 & 55 & 27 & 28 \\
119 & 54.5 & 31 & 23.5 \\
203 & 55.7 & 21.6 & 34.1 \\
207 & 55.8 & 26 & 29.8 \\
210 & 55.8 & 17.8 & 38 \\
\hline
\end{tabular}

Table 5 PSD criteria for evaluating $60 \mathrm{~Hz}$ noise suppression.

\begin{tabular}{lllll}
\hline \multirow{2}{*}{ ECG } & \multirow{2}{*}{ Amplitude A A } & \multicolumn{3}{c}{ PSD at 60 Hz (dB/Hz) } \\
\cline { 3 - 5 } & & $\begin{array}{l}\text { Before } \\
\text { filtering }\end{array}$ & $\begin{array}{l}\text { After } \\
\text { filtering }\end{array}$ & Attenuation \\
\hline \multirow{2}{*}{117} & 20 & 23 & -30 & 53 \\
& 100 & 36 & -30 & 66 \\
119 & 20 & 23 & -23 & 46 \\
& 100 & 36 & -22 & 58 \\
203 & 20 & 23 & -23 & 46 \\
& 100 & 38 & -23 & 61 \\
207 & 20 & 23 & -25 & 48 \\
& 100 & 36 & -25 & 61 \\
210 & 20 & 23 & -30 & 53 \\
& 100 & 36 & -30 & 66 \\
\hline
\end{tabular}

Table 6 COR \& PRD criteria for evaluating noise suppression.

\begin{tabular}{lllll}
\hline \multirow{2}{*}{ ECG } & \multicolumn{2}{c}{ Baseline wander } & \multicolumn{2}{c}{$60 \mathrm{~Hz}$ interferences } \\
\cline { 2 - 5 } & COR (\%) & PRD (\%) & COR $(\%)$ & PRD (\%) \\
\hline 117 & 100 & 0 & 100 & 0,152 \\
119 & 100 & 0 & 100 & 0,290 \\
203 & 100 & $1,44 \times 10^{-13}$ & 100 & 0,691 \\
207 & 100 & $1,93 \times 10^{-13}$ & 100 & 0,221 \\
210 & 100 & $1,57 \times .10^{-13}$ & 100 & 0,230 \\
\hline
\end{tabular}


For the $60 \mathrm{~Hz}$ power line noise, we see that it is completely removed regardless of its magnitude, and we get the sinusoidal shape of the noise. The PSD after filtering has the same value of each signal.

It is established in Ref. [14] that if the PRD value is between $0 \%$ and $9 \%$, the quality of the reconstructed signal is either"very good" or "good", whereas if the value is greater than $9 \%$ its quality group cannot be determined. As we are strictly interested in very good and good reconstructions, it is taken that the PRD value, as measured with Eq. (10), must be less than $9 \%$. Table 6 shows that our method performs a COR equal to $100 \%$, and a PRD equal to $0.691 \%$ at most. This reflects that the two signals, before and after denoising, are identical.

\section{Conclusion}

In this work, we have presented a new approach based on discrete wavelet decomposition for denoising the ECG signals. The results illustrate that the DWT is an efficient technique to filter noises without altering a real morphology ECG signals because the duration, amplitude and shape of the $\mathrm{P}$ wave, QRS complex and $\mathrm{T}$ wave are not modified. This technique applies to all types of ECG signals, whether they are normal or presenting arrhythmias. Hence, this process allows cardiovascular experts to make a proper analysis. It can be integrated in automatic ECG analysis systems.

\section{References}

[1] Addison, S.P. 2005. "Wavelet Transforms and the ECG." Physiol. Meas. 26:R155-99.

[2] Thakor, N.V., Webster, J.G., and Thompkins, W.J. 1984. "Estimation of QRS Complex Power Spectra for Design of a QRS Filter." IEEE Trans. Biomed. Eng. 31: 702-5.
[3] Thakor, N.V., and Zhu, Y.S. 1991. "Applications of Adaptive Filtering to ECG Analysis: Noise Cancellation and Arrhythmia Detection." IEEE Trans. Biomed. Eng. 38 (8): 785-94.

[4] Weng, B., Blanco-Velasco,M.,and Barner, K.E. 2006. "Baseline Wander Correction in ECG by the Empirical Mode Decomposition." InProceedings of the IEEE 32nd Annual Northeast Bioengineering Conference, 135-6.

[5] Taouli, S.A.,and Reguig, F.B. 2010. "Noise and Baseline Wandering Suppression of ECG Signals by Morphological Filter." Journal of Medical Engineering \& Technology34 (2): 87-96.

[6] Zahhad, M.A., Ahmed, S. M.,and Zakaria, A. 2011. "ECG Signal Compression Technique Based on Discrete Wavelet Transform and QRS-Complex Estimation." Signal Processing : An International Journal (SPIJ) 4 (2): 138-60.

[7] Mallat, S. 1989. "A Theory for Multiresolution Signal Decomposition: The Wavelet Representation." IEEE Trans. on Patt. Anal.and Mach. Intell.11 (7): 674-93.

[8] MIT-BIHd://www.physionet.org/physiobank/database/mi $\mathrm{tdb} /$.

[9] Zidelmal, Z., Amirou, A., Adnaneb, M., and Belouchranib, A. 2012. "QRS Detection Based on Wavelet Coefficients." Computer Methods and Programs in Biomedicine 107(2012): 490-6.

[10] Pan, J.,and Tompkins, W. J. 1985. "A Real Time QRS Detection Algorithm." IEEE Trans. Biomed. Eng. 32: 230-6.

[11] Rahul, D.,and Mehrotra, R. K. 2013. "Application of DSP to Remove Noise from ECG Signal."International Journal of Engineering Research \& Technology (IJERT) 2 (6): 2421-8.

[12] Pan, N., Mang, V., Un, M.P., and Hang, P.S. 2007. "Accurate Removal of Baseline Wander in ECG Using Empirical Mode Decomposition." In Proceedings of NFSI \& ICFBI, 177-80.

[13] Ji, T.Y., Lu, Z., Wu, Q.H., and Ji, Z. 2008. "Baseline Normalisation of ECG Signals Using Empirical Mode Decomposition and Mathematical Morphology." ELECTRONICS LETTERS 44 (2): 82-3.

[14] Zigel, Y., Cohen, A., and Katz, A. 2000. "The Weighted Diagnostic Distortion (WDD) Measure for ECG Signal Compression." IEEE Trans. Biomed. Eng.47: 1422-30. 